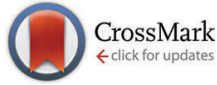

Cite this: Phys. Chem. Chem. Phys., 2014, 16, 23843

Received 15th August 2014, Accepted 11th September 2014

DOI: $10.1039 / c 4 c p 03659 j$

www.rsc.org/pccp

\section{Investigations into the nature of spontelectrics: nitrous oxide diluted in xenon}

\author{
Andrew Cassidy, ${ }^{a}$ Oksana Plekan, $\dagger^{a}$ Jack Dunger, ${ }^{a}$ Richard Balog, ${ }^{a}$ \\ Nykola C. Jones, ${ }^{b}$ Jérôme Lasne, ${ }^{C}$ Alexander Rosu-Finsen, ${ }^{C}$ Martin R. S. McCoustra ${ }^{c}$ \\ and David Field*a
}

\begin{abstract}
The recent discovery of a new class of solids displaying bulk spontaneous electric fields as high as $10^{8} \mathrm{~V} \mathrm{~m}^{-1}$, so-called 'spontelectrics', poses fundamental and unresolved problems in solid state physics. The purpose of the present work is to delve more deeply into the nature of the interactions which give rise to the spontelectric effect in films of nitrous oxide $\left(\mathrm{N}_{2} \mathrm{O}\right)$, by observing the variation of the spontaneous field as the $\mathrm{N}_{2} \mathrm{O}$ molecules are physically removed from one another by dilution in Xe. Data, obtained using the ASTRID storage ring, are presented for films diluted by factors $\xi=\mathrm{Xe} / \mathrm{N}_{2} \mathrm{O}$ of 0.9 to 67 , at deposition temperatures of $38 \mathrm{~K}, 44 \mathrm{~K}$ and $48 \mathrm{~K}$, where films are laid down by deposition from a gas mixture. Results show that the spontelectric field decreases as $\xi$ increases and that at $\xi=67$ for $44 \mathrm{~K}$ deposition, the spontelectric effect is absent. Reflection-absorption infrared spectroscopy (RAIRS) data are also reported, providing insight into the structure of $\mathrm{Xe} / \mathrm{N}_{2} \mathrm{O}$ films and specifically showing that $\mathrm{N}_{2} \mathrm{O}$ remains dispersed in the $\mathrm{Xe} / \mathrm{N}_{2} \mathrm{O}$ films prepared here. A simplified theoretical model is developed which illustrates that electric fields can be understood in terms of dilution-dependent dipole orientation. This model is used to reproduce experimental data up to an average molecular separation, $s$, of $\geq 1.25 \mathrm{~nm}$ apart, $\sim 4$ times that associated with pure solid $\mathrm{N}_{2} \mathrm{O}$. The disappearance of the spontelectric effect at larger average distances of separation, between $s=1.25 \mathrm{~nm}$ and $s=1.75 \mathrm{~nm}$, is a phenomenon which cannot be described by any existing model but which shows that dipole-dipole interactions are an essential ingredient for the creation of the spontelectric state.
\end{abstract}

\section{Introduction}

Spontelectrics form a new group of solid state materials, ${ }^{1-7}$ whose salient characteristic is that they exhibit a spontaneous electric field within the bulk of the solid. The spontelectric state is unique in the physics of solids and shows characteristics quite distinct from any other known solids, such as the ferroelectric state of matter. ${ }^{1}$ The fundamental interactions which govern the nature of the spontelectric state and the processes whereby the spontelectric state is created remain however elusive. It is the purpose of this paper to shed additional light on the nature of the spontelectric state. Experimental results reported here for the spontelectric material $\mathrm{N}_{2} \mathrm{O}$ diluted in Xe, further characterize the spontelectric state and place additional

\footnotetext{
${ }^{a}$ Department of Physics and Astronomy, Aarhus University, DK-8000 Aarhus C, Denmark. E-mail: dfield@phys.au.dk

${ }^{b}$ ISA, Department of Physics and Astronomy, Aarhus University, DK-8000 Aarhus C, Denmark

${ }^{c}$ Institute of Chemical Sciences, Heriot-Watt University, Riccarton, EH14 4AS Edinburgh, UK

$\dagger$ Present address: Sincrotrone Trieste, S.C.p.A. di Interesse Nazionale, 34149 Basovizza, Trieste, Italy.
}

constraints on any future $a b$ initio models of the structure or dynamical models of the creation of this state.

For the present, it is evident that the properties of spontelectrics arise through collective effects resulting in the orientation of permanent dipole moments of the constituent species. However it remains unclear whether dipole-dipole interactions between the active species are an essential ingredient of the physics which leads to this orientation. From hereon, dipole orientation refers to the tendency of dipoles to align in energetically unfavourable configurations in which $\delta+$ lies on average closer to another $\delta+$ and correspondingly $\delta-$ to $\delta-$, where $\delta+$ and $\delta$ - refer to opposite dipolar ends of the species.

Here we address the following questions: (i) how does the degree of dipole orientation change as the spontelectric species are removed physically from one another by dilution in a solid matrix of an inert gas? (ii) Is there some average physical separation between species at which the spontelectric effect can no longer be observed? The latter is the major qualitative aim of the present work and essentially tests the hypothesis that dipole-dipole interactions are necessary to the spontelectric effect - and it is found that they are necessary.

Questions (i) and (ii) are addressed by studying films of $\mathrm{N}_{2} \mathrm{O}$ diluted in Xe in which, with increasing dilution, the $\mathrm{N}_{2} \mathrm{O}$ 
molecules are increasingly further apart and are thus more weakly interacting. $\mathrm{N}_{2} \mathrm{O}$ is known to exhibit the spontelectric effect and it has been investigated in considerable detail in earlier work. ${ }^{1,2,4}$ Indeed it was the first species which was shown to exhibit this effect. ${ }^{2}$

A parameterized model is developed which adequately addresses question (i) posed above. This model quantitatively reproduces observations of a reduction in the spontaneous electric field in films of $\mathrm{N}_{2} \mathrm{O}$ with increasing dilution in Xe. The answer to question (ii), as we have indicated, is that when the dilution and the corresponding average separation of dipolar species is sufficient, there is no evidence of a spontaneous field within the film. This occurs for a factor of dilution of between 26 and 67 for $\mathrm{N}_{2} \mathrm{O}$ in Xe deposited at $44 \mathrm{~K}$. We estimate the critical separation of $\mathrm{N}_{2} \mathrm{O}$ species for the loss of the spontelectric effect to lie between 1.25 and $1.75 \mathrm{~nm}$.

The characteristics of spontelectrics in pure films have recently been reviewed in ref. 1 . In brief, the spontelectric effect is experimentally characterized by the following properties:

- On forming a film by gas phase deposition under ultrahigh vacuum (UHV) of a spontelectric material, a polarization potential, typically of several volts, appears spontaneously on the surface of the film.

- This potential, linearly proportional to the thickness of the film, may be either positive or negative and gives rise to a corresponding spontelectric field in the film.

- The spontelectric field depends on both the nature of the material which is deposited and on the temperature at which the film is deposited. The field for any substance is in general less for higher temperatures (save for methyl formate deposited at $\geq 80 \mathrm{~K})$.

- At greater than a certain temperature of deposition, no spontelectric effect can be observed.

- Warming of a spontelectric film causes at first little change in the spontaneous potential on the surface of the film, but a critical temperature is reached at which the spontelectric effect decays abruptly.

- The nature of the substrate surface, upon which spontelectric films are deposited, has essentially no bearing on the value of the spontelectric field within the film. ${ }^{7}$

Materials which show the spontelectric effect are very diverse $^{1}$ and include species such as nitrous oxide, propane, toluene or chlorotrifluoromethane. Films of material may contain electric fields which can exceed $10^{8} \mathrm{~V} \mathrm{~m}^{-1}$, noting that breakdown fields of solids lie between $10^{8}$ to $10^{9} \mathrm{~V} \mathrm{~m}^{-1}$. These fields are spontaneous, created without any external stimulus, such as an applied electric field; hence the term 'spontelectric'.

Spontelectric films constitute a form of the solid state in which both non-linear effects and non-local, collective effects operate. The spontelectric effect is non-linear in the sense that in the steady state the degree of dipole orientation and the spontelectric field are related in a manner such that one depends upon the other: that is, the degree of dipole orientation depends upon an expansion in powers of the local field at any dipole and the local field itself depends on the degree of dipole orientation (see for example ref. 1 and eqn (1) and (2) in Section 3.1).
The spontelectric effect is non-local since it is a bulk phenomenon which cannot be described in terms of local interactions alone. Rather, the spontelectric field permeates the entire thickness of the film and assumes a value which is due to a combination of interactions which extend both over the entire film as well as through pair-wise or few-body local intermolecular interactions. In this connection, we observe experimentally that in general a certain thickness of the sample is required before the spontelectric effect develops. It is found for example that isoprene deposited at $70 \mathrm{~K}$ requires $>50$ monolayers $(\mathrm{ML})$ or toluene at $75 \mathrm{~K}$ requires $>100 \mathrm{ML}$ to develop a substantial electric field (Fig. 4 and 5 of ref. 1). In this respect the spontelectric effect is unique in solid state physics since the steady-state structures with which it is associated depend upon effects of long range feedback. This feedback results in such counter-intuitive behaviour as the increase in dipole orientation, seen in methyl formate, with increasing temperatures of deposition above $80 \mathrm{~K}^{5}$

Our current qualitative understanding of the spontelectric phenomenon is as follows. On deposition of a dipolar gas to form a film, species adjust such that dipoles should tend to be configured on average in head to tail configurations, that is, $\delta+$ to $\delta-$. However thermal fluctuations may give rise to temporary configurations in which are found local average excess of $\delta+$ close to $\delta+$ rather than $\delta$ - and correspondingly an excess of $\delta$ - close to $\delta-$, that is, net dipole orientation. Here, fluctuations play the role of the externally applied field in the standard description of dielectric polarization.

Such dipole aligned configurations are evidently accompanied by a local energy deficit due to repulsions between molecules. These configurations will create a fleeting and spatially localised electric field which tends to oppose the orientation. There are now two possibilities. In the first place, the effect may remain a local fluctuation which continually forms, dies away and is resurrected, with dipole orientation averaging to zero over time and space. In the second, given a large enough local fluctuation, there may be a macroscopic switch to a dipole orientation opposing that set up through thermal fluctuation. This opposing dipole orientation then propagates throughout the system via the corresponding opposing electric field. This is posited to occur since, outside the immediate region of the fluctuation, there is instantaneously no, or less, net dipole orientation. In this model, the opposing field, in a region exterior to the fluctuation, sets up dipole orientation in the opposite sense to the fluctuation itself. It is dipole orientation in this opposite sense which gives rise to the observed spontelectric field. Note that the film is not itself an isolated system but is fed by an external cooling system. Moreover the spontelectric state would not appear to be a minimum energy state, but rather a metastable state into which the system becomes lodged in its exploration of the phase space on its journey towards the lowest energy configuration. Having entered the spontelectric state, there are substantial local barriers to the equilibrium state, much as there are in, say, amorphous water ice whose minimum energy configuration, unattainable on any practical timescale at low temperature, is hexagonal ice. 
With regard to this qualitative model of the generation of spontelectrics, an electric field must of course be described by two regions of established potential and, within the above model, these locations may instantaneously be within a region of a fluctuation and outside of it. As an important, perhaps crucial, special case, one of these regions may be the surfacevacuum interface, the nature of which may determine the sign of the spontelectric field. Thus $\mathrm{N}_{2} \mathrm{O}$ at the surface is configured with the terminal $\mathrm{N}$ atom, which is $\delta+$, pointing into the vacuum whereas, for example, $\mathrm{CF}_{3} \mathrm{Cl}$ is configured with the $\mathrm{Cl}$ atom pointing outwards, giving a spontelectric field of the opposite sign. ${ }^{6}$

In addition, the dynamical model put forward above implies that the presence of thermal fluctuations alone may be sufficient in causing the spontelectric phenomenon. However the dynamics of the constituent species will be modulated by local dipoledipole interactions. Our present work shows that such dipoledipole interactions play a fundamental role in creating the spontelectric effect and that a model based solely upon independent fluctuations of the active species is insufficient.

\section{Experimental method and results}

\subsection{The experimental method}

Surface potential measurements were performed on the SGM2 beamline at the ASTRID synchrotron storage ring at Aarhus University (www.isa.au.dk). The experimental chamber operates under ultra-high vacuum conditions, with a base pressure of $\sim 2 \times 10^{-10}$ mbar. Films were grown on a polycrystalline $\mathrm{Au}$ substrate which was cooled via a closed cycle He cryostat to a minimum temperature of $38 \mathrm{~K}$. Before film growth the substrate was cleaned by annealing to $700 \mathrm{~K}$ using a cartridge heater. Background vapour deposition was used to grow all films with the substrate fixed at a chosen temperature.

Throughout, film thicknesses are described in monolayers (ML), where one ML of coverage was calibrated from temperature programmed desorption (TPD) experiments. A single ML of nitrous oxide (or xenon) refers to the number of species required to saturate adsorption sites on the polycrystalline Au surface. The residual gas pressure in the chamber during dosing was measured using a quadrupole mass spectrometer, so that a given background pressure could be correlated to a dosing rate. The residual gas pressure method was used to fine-tune actual final dosing rates while dosing gas mixtures for the creation of diluted films. Dilution ratio is defined here as the effective number of ML of Xe divided by the number of ML of nitrous oxide.

Gas mixtures were prepared in an adjacent gas-line, also under UHV, before admission to the experimental chamber. Nitrous oxide ( $\geq 99.998 \%)$ and xenon $(99.99 \%)$ were used as received from Sigma Aldrich. Nitrous oxide was loaded into an initially isolated gas-line, using a continuous flow of gas, until the pressure required to maintain the dosing flux for a required dose rate (ML $\mathrm{min}^{-1}$ ), known from our calibration experiments, was achieved. Xenon gas was then mixed into the continuous flow of nitrous oxide until the required dilution, again estimated from initial calibration experiments, was achieved. Thus the gases were pre-mixed in a known ratio in a flowing system. This flow was then exposed to the cold substrate in the experimental chamber for an appropriate time to create a mixed film corresponding to any selected dilution. Films were built up by the sequential deposition of layers of the xenon-nitrous oxide matrix. The rate of deposition was typically 5 to $10 \mathrm{ML} \mathrm{min}^{-1}$. In earlier work we have tested the hypothesis that values of surface potential may be dependent on deposition rate, by varying the rate through a factor of ten. No effects were found and no further checks of this sort were carried out here.

As far as was practically possible, the nitrous oxide fluence, in terms of number of ML, was kept constant for all experiments at both different temperatures and dilutions. Thus for any deposition step, a fluence of $\sim 50 \mathrm{ML}$ of nitrous oxide was deposited, with the xenon fluence adjusted to give the desired degree of dilution. This technique ensured that for separate experiments performed, say, with increasing dilution, the layer of material contained always the same total number of $\mathrm{N}_{2} \mathrm{O}$ molecules, but separated from one another by an increasing distance by intervening Xe atoms. Thus the column density of $\mathrm{N}_{2} \mathrm{O}$ remains approximately constant in all experiments whereas the column length, that is the film thickness, becomes increasingly greater with greater dilution. For the present we view $\mathrm{N}_{2} \mathrm{O}$ as remaining mixed with $\mathrm{Xe}$, rather than separated from Xe in the deposited film. We return to this point in detail below. The quoted absolute film thicknesses are accurate to within $20-30 \%$, but relative thicknesses for any experiment for a specific dilution and deposition temperature are accurate to within a few per cent.

Details of the experimental method for measurement of film surface potentials have been described elsewhere. ${ }^{1}$ In brief, using synchrotron radiation from ASTRID, low energy electrons were produced by photoionization of Ar gas at $\sim 5 \mathrm{meV}$ above threshold $(78.56 \mathrm{~nm})$ in a source chamber and with an energy spread given by that of the photoionizing radiation, set here to be $\sim 1.5 \mathrm{meV}$. These low energy electrons were extracted, via a series of lenses, and formed into a beam. The potentials on the film surfaces are estimated using the principle that an electron beam formed at the potential of the centre of the photoionization chamber can just reach another point, for example the surface of a film or of clean gold, at the same potential, potential barriers apart. Thus if electrons are formed at a known potential, the potential of their destination can be measured by the bias that must be applied, either to their point of formation or their destination, such that a current just begins to flow.

The spontaneous potential appearing on the surface of spontelectric films is measured by applying such a bias potential to the system and effectively adjusting this bias in order to null the current. Since electrons are formed at nominally zero volts with an energy of $\sim 5 \mathrm{meV}$, the beam should therefore just be able to reach a clean $\mathrm{Au}$ target when the target is itself at this same nominal zero (less $5 \mathrm{mV}$ ). If the target were covered with a film of spontelectric material and its surface were not at zero but rather, say, at +5 volts, due to the spontaneous formation of an electric field within the film, then in order to ensure that the electrons only just reach the target, it would be necessary to bias the target, or the point of formation of the electrons, by 5 volts. 
Measurement of the bias required to locate the onset of a measureable current $(\sim 2 \mathrm{fA})$ therefore gives the potential on the surface of the film. A small correction is made to establish a true zero difference between the clean gold substrate and the potential of formation of the electrons. This arises from the difference between the work function of the gold substrate and the graphite-covered walls of the photoionization chamber in which electrons are formed. This difference is measured for each series of experiments; a typical value of $\sim 0.25 \mathrm{~V}$ is found, in agreement with standard figures. As emphasized in earlier work, we interrogate the material while perturbing it to a minimum. This was ensured by using very small currents, typically of 100-200 fA, in order not to reduce significantly the spontaneous polarization of the film surface or introduce negative charging. These low currents were detected using a Keithley 6340 femtoammeter.

\subsection{Results}

Experimental data are shown in Fig. 1 and 2 for deposition of diluted films at temperatures of $44 \mathrm{~K}$ and $48 \mathrm{~K}$ respectively. Fig. 3 shows data at $38 \mathrm{~K}, 44 \mathrm{~K}$ and $48 \mathrm{~K}$ with dilutions of 2.1, 2.2 and 2.8 respectively. Corresponding voltages per $\mathrm{ML}$ of $\mathrm{N}_{2} \mathrm{O}$ added are shown in Tables 1-3.

\subsection{The nature of $\mathrm{Xe} / \mathrm{N}_{2} \mathrm{O}$ films; reflection-absorption infrared spectroscopy studies}

As mentioned above, we have taken the view that films laid down from gas mixtures of $\mathrm{Xe}$ and $\mathrm{N}_{2} \mathrm{O}$ are themselves mixed and do not segregate into pure $\mathrm{Xe}$ and $\mathrm{N}_{2} \mathrm{O}$ phases. The only evidence for this is that previous studies ${ }^{8,9}$ have shown that at low concentrations, that is, $\xi=729$ to 1000 and temperatures of 16 to $45 \mathrm{~K}, \mathrm{~N}_{2} \mathrm{O}$ does not segregate from Xe matrices and remains in the form of monomers. However a simple argument, given below, shows the experimental data presented in Fig. 1 to 3 illustrate that Xe and $\mathrm{N}_{2} \mathrm{O}$ remain mixed for the much lower values of dilution used here.

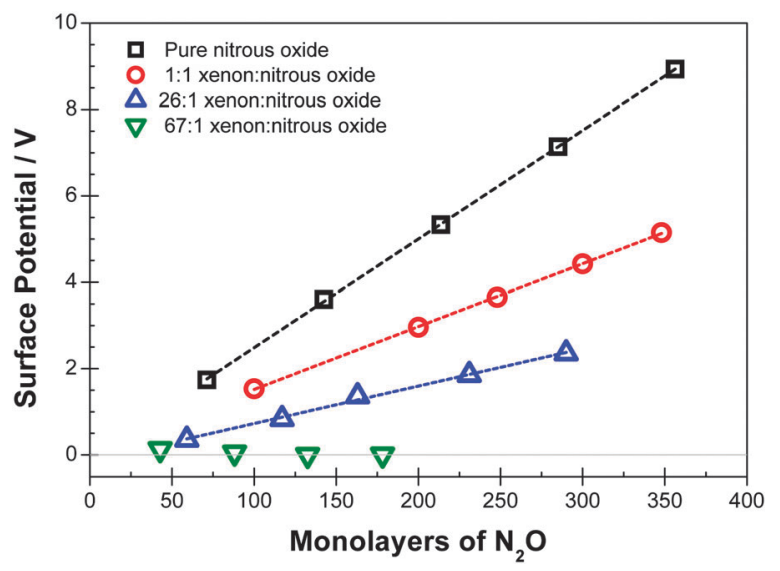

Fig. 1 Surface potentials measured for mixtures of $\mathrm{N}_{2} \mathrm{O}$ and $\mathrm{Xe}$ deposited at $44 \mathrm{~K}$ for the dilutions shown. The thickness of the layer is expressed as the equivalent number of $\mathrm{ML}$ of $\mathrm{N}_{2} \mathrm{O}$ deposited, excluding Xe. Note the absence of a spontelectric effect at a dilution, $\xi$, of 67 .

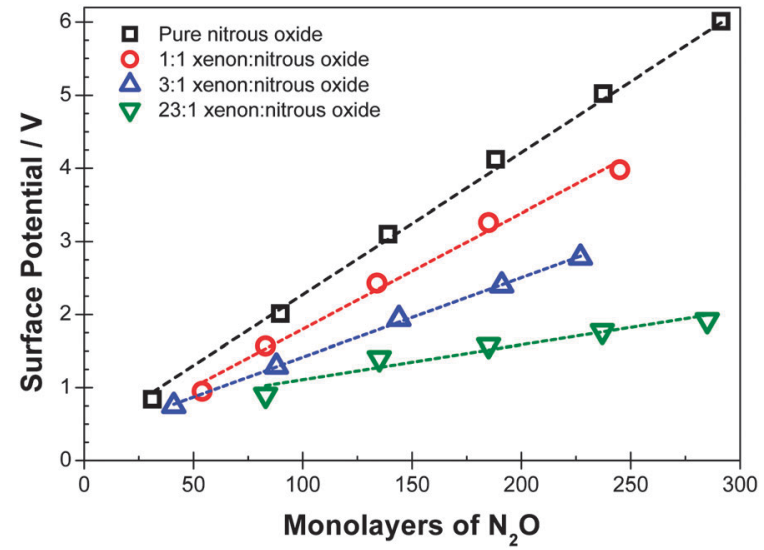

Fig. 2 Surface potentials measured for mixtures of $\mathrm{N}_{2} \mathrm{O}$ and Xe deposited at $48 \mathrm{~K}$ for the dilutions shown. The thickness of the layer is expressed as the equivalent number of $\mathrm{ML}$ of $\mathrm{N}_{2} \mathrm{O}$ deposited, excluding $\mathrm{Xe}$.

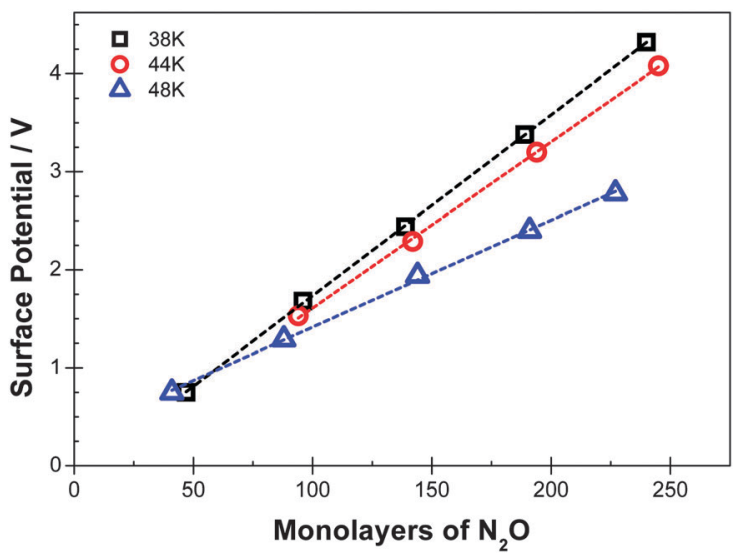

Fig. 3 Surface potentials measured for mixtures of $\mathrm{N}_{2} \mathrm{O}$ and $\mathrm{Xe}$ deposited at $38 \mathrm{~K}, 44 \mathrm{~K}$ and $48 \mathrm{~K}$ at dilutions of $2.1,2.2$ and 2.8 respectively (see Table 3). The thickness of the layer is expressed as the equivalent number of $\mathrm{ML}$ of $\mathrm{N}_{2} \mathrm{O}$ deposited, excluding Xe.

Table 1 Values of $m V$ of potential added per equivalent $M L$ of $\mathrm{N}_{2} \mathrm{O}$ laid down at $44 \mathrm{~K}$, for dilutions, $\xi=\mathrm{Xe} / \mathrm{N}_{2} \mathrm{O}$, as shown

\begin{tabular}{lll}
\hline $\begin{array}{l}\text { Deposition } \\
\text { temperature }(\mathrm{K})\end{array}$ & $\begin{array}{l}\text { Dilution } \\
\xi: \mathrm{Xe} / \mathrm{N}_{2} \mathrm{O}\end{array}$ & $\begin{array}{l}\mathrm{mV} \text { per equivalent } \\
\mathrm{ML} \text { of } \mathrm{N}_{2} \mathrm{O}\end{array}$ \\
\hline 44 & 0 & 25 \\
44 & 1.0 & 15 \\
44 & 26 & 9 \\
44 & 67 & 0
\end{tabular}

It is known from other work that deposition of $\mathrm{N}_{2} \mathrm{O}$ onto a Xe layer or deposition of Xe on top of a $\mathrm{N}_{2} \mathrm{O}$ layer has no measureable effect on the spontelectric field in the $\mathrm{N}_{2} \mathrm{O} .{ }^{1}$ Thus segregation into pure Xe and $\mathrm{N}_{2} \mathrm{O}$ phases in the present work would result in a lack of any dependence of the spontelectric field on the value of the dilution, in clear contrast to observation. This provides conclusive evidence that Xe and $\mathrm{N}_{2} \mathrm{O}$ do not segregate in the films studied here.

This qualitative result still leaves open the question of clustering in mixed films. For lower values of $\xi$ it is inevitable 
Table 2 Values of $\mathrm{mV}$ of potential added per equivalent $\mathrm{ML}$ of $\mathrm{N}_{2} \mathrm{O}$ laid down at $48 \mathrm{~K}$, for dilutions, $\xi=\mathrm{Xe} / \mathrm{N}_{2} \mathrm{O}$, as shown

\begin{tabular}{lll}
\hline $\begin{array}{l}\text { Deposition } \\
\text { temperature }(\mathrm{K})\end{array}$ & $\begin{array}{l}\text { Dilution } \\
\xi: \mathrm{Xe} / \mathrm{N}_{2} \mathrm{O}\end{array}$ & $\mathrm{mV}$ per equivalent \\
\hline 48 & 0 & $\mathrm{ML}$ of $\mathrm{N}_{2} \mathrm{O}$ \\
48 & 0.9 & 21 \\
48 & 2.8 & 16 \\
48 & 23 & 72
\end{tabular}

Table 3 Values of $\mathrm{mV}$ of potential added per equivalent $\mathrm{ML}$ of $\mathrm{N}_{2} \mathrm{O}$ laid down at $38 \mathrm{~K}, 44 \mathrm{~K}$ and $48 \mathrm{~K}$, for dilutions, $\xi=\mathrm{Xe} / \mathrm{N}_{2} \mathrm{O}$, as shown. We note that discrepancies between data at $44 \mathrm{~K}$ for $\xi=1$ and $\xi=2.2$ in Tables 1 and 3 lie within the $20-30 \%$ accuracy attributed to absolute values of layer thickness

\begin{tabular}{lll}
\hline $\begin{array}{l}\text { Deposition } \\
\text { temperature }(\mathrm{K})\end{array}$ & $\begin{array}{l}\text { Dilution } \\
\xi: \mathrm{Xe} / \mathrm{N}_{2} \mathrm{O}\end{array}$ & $\mathrm{mV}$ per equivalent \\
\hline 38 & 2.1 & 18 \\
44 & 2.2 & 16 \\
48 & 2.8 & 12
\end{tabular}

that $\mathrm{N}_{2} \mathrm{O}$ species must find themselves juxtaposed to form dimers, trimers etc. In order to investigate this more subtle effect on film structure with dilution, experiments were performed using reflection-absorption infrared spectroscopy (RAIRS) at Heriot-Watt University. ${ }^{10}$ These confirm that nitrous oxide molecules were dispersed in the film on deposition of the gas mixture and did not segregate from the Xe matrix when laid down from a gas mixture. Experiments also demonstrate the juxtaposition, and thus association, to form dimers, trimers etc. of $\mathrm{N}_{2} \mathrm{O}$ within Xe.

RAIRS experiments were performed as follows. Mixtures with $\xi=5$ were prepared in a gas line maintained under vacuum and in dark conditions to avoid photodissociation. The mixing ratio was determined using a capacitance manometer and confirmed using a quadrupole mass spectrometer (Hiden Analytical Ltd, HAL301) inside the chamber. Thick films were grown by background dosing of the $\mathrm{Xe}-\mathrm{N}_{2} \mathrm{O}$ mixture onto a copper substrate coated with $300 \mathrm{~nm}$ silica. Film thicknesses in ML were determined from the TPD of $\mathrm{N}_{2} \mathrm{O}$ from the silica-coated substrate. RAIR spectra were recorded over the range $400-4000 \mathrm{~cm}^{-1}$ at a resolution of $1 \mathrm{~cm}^{-1}$ using a Fourier-transform infrared spectrometer (Varian 670-IR) set at a grazing incidence of $75^{\circ}$ with respect to the normal of the substrate. We note that the spontelectric effect does not depend on the nature of the substrate on which the films are deposited. ${ }^{7}$ Therefore the RAIRS results on a silica substrate remain relevant to the surface potential measurements on polycrystalline Au performed at the ASTRID laboratory.

Initially a $\xi=5$ film containing the equivalent of $48 \mathrm{ML}$ of $\mathrm{N}_{2} \mathrm{O}$ was deposited at $20 \mathrm{~K}$ on silica and monitored with RAIRS. This film gives rise to a single partially resolved set of features around $2220 \mathrm{~cm}^{-1}$ belonging to the adsorbate, over the 400$4000 \mathrm{~cm}^{-1}$ range investigated. Fig. 4 shows this set of features, where the lowest frequency, centered at $2214.5 \mathrm{~cm}^{-1}$, corresponds to the NN stretching mode of the isolated $\mathrm{N}_{2} \mathrm{O}$ monomer in Xe as discussed below. ${ }^{8,9}$ Spectra of films deposited at $29 \mathrm{~K}$ and $38 \mathrm{~K}$ are also shown in Fig. 4. We note that TPD experiments on Xe films adsorbed on silica show that Xe does

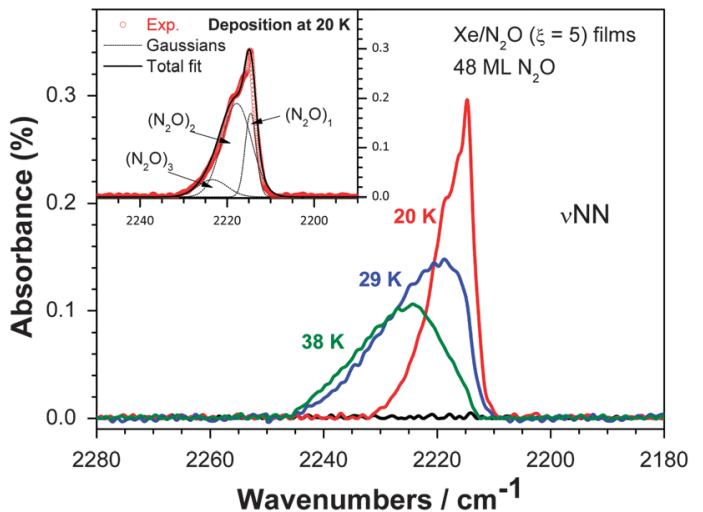

Fig. $4 \nu$ NN region of the RAIR spectra of the silica substrate before (black line) and after deposition of a $\mathrm{Xe}-\mathrm{N}_{2} \mathrm{O}(\xi=5)$ solid mixture equivalent to $48 \mathrm{ML} \mathrm{N} \mathrm{O}_{2} \mathrm{O}$ at $20 \mathrm{~K}$ (red line), $29 \mathrm{~K}$ (blue) or $38 \mathrm{~K}$ (green). The inset presents the experimental spectrum of the mixture as deposited at $20 \mathrm{~K}$ (red circles). This spectrum was fitted with gaussian functions representing the contributions of $\mathrm{N}_{2} \mathrm{O}$ monomer, dimer and trimer (dotted lines); the overall fit is shown with a black line in the inset.

not stick efficiently to silica above $42 \mathrm{~K}$, precluding deposition studies at higher temperatures.

Fig. 4 shows that as the deposition temperature is raised, the intensity on the low wavenumber side of the band (2210$2220 \mathrm{~cm}^{-1}$ ) decreases, while intensity is gained on the high wavenumber side $\left(2220-2245 \mathrm{~cm}^{-1}\right)$. A similar change of the RAIR spectra is observed when the film is deposited at $19 \mathrm{~K}$ and annealed to $49 \mathrm{~K}$, just below the onset of Xe desorption at $50 \mathrm{~K}$. Significantly however, the $\nu \mathrm{NN}$ bands of a pure $\mathrm{N}_{2} \mathrm{O}$ multilayer, characterized by bands at 2260 and $2235 \mathrm{~cm}^{-1}$ in RAIR spectra recorded on non-metallic surfaces, ${ }^{11-13}$ are absent from the spectra in Fig. 4 or in similar spectra for annealing of mixtures up to $49 \mathrm{~K}$ (not shown). This demonstrates the major result of the RAIRS study that $\mathrm{N}_{2} \mathrm{O}$ does not separate from Xe to form a pure solid layer but remains dispersed within the xenon matrix for $\xi=5$ and temperatures between $20 \mathrm{~K}$ and $38 \mathrm{~K}$ for deposition, and $49 \mathrm{~K}$ for annealing.

To obtain the relative contributions from $\mathrm{N}_{2} \mathrm{O}$ monomer, dimer etc., the band centered at $2220 \mathrm{~cm}^{-1}$ for both deposition data (Fig. 4) and annealing data was fitted using the addition of varying proportions of four bands in the form of gaussian functions, where each gaussian centre corresponds to an absorption frequency of $\left(\mathrm{N}_{2} \mathrm{O}\right)_{x}$, where $x$ ranges from 1-4. For the monomer in a Xe matrix, the relevant frequency is $2214.5 \mathrm{~cm}^{-1}$. $^{8,9}$ To the best of our knowledge, the absorption frequencies for $x=2,3$ and 4 in a Xe matrix are not available and we set out to estimate them here. Kudoh et al. ${ }^{14}$ isolated $\mathrm{N}_{2} \mathrm{O}$ in an argon matrix and observed the monomer band at $2218.6 \mathrm{~cm}^{-1}$. From this value, the difference between the matrix shifts in Xe and Ar for the $\nu \mathrm{NN}$ band of $\mathrm{N}_{2} \mathrm{O}$ is taken to be $-4.1 \mathrm{~cm}^{-1}$ in Xe relative to Ar. This gives access to estimates of dimer and trimer band frequencies in Xe since the bands of $\mathrm{N}_{2} \mathrm{O}$ dimer and trimer in $\mathrm{Ar}$ have been reported. ${ }^{14,15}$ Given our estimated matrix shift difference of $-4.1 \mathrm{~cm}^{-1}$, frequencies of 2218.0 and $2223.4 \mathrm{~cm}^{-1}$ may be assigned to $\mathrm{N}_{2} \mathrm{O}$ dimers and trimers in Xe respectively, using 
the available Ar data. In addition, the $\mathrm{N}_{2} \mathrm{O}$ tetramer has been observed in a supersonic helium jet expansion with a strong Q-branch at $2237.43 \mathrm{~cm}^{-1} \cdot{ }^{16}$ Given the gas phase nature of the jet expansion environment, a matrix shift for Xe of $\Delta \nu($ gas $-\mathrm{Xe})=$ $8.9 \mathrm{~cm}^{-1}$ for the $\nu \mathrm{NN}$ band of $\mathrm{N}_{2} \mathrm{O}^{9}$ may be applied and gives an expected band at $2228.5 \mathrm{~cm}^{-1}$ for $\left(\mathrm{N}_{2} \mathrm{O}\right)_{4}$ in Xe, the value therefore assigned to this tetramer.

Adopting the above values for vibrational frequencies of $\left(\mathrm{N}_{2} \mathrm{O}\right)_{x}$, respectively $2214.5 \mathrm{~cm}^{-1}, 2218 \mathrm{~cm}^{-1}, 2223.4 \mathrm{~cm}^{-1}$ and $2228.5 \mathrm{~cm}^{-1}$, and FWHMs of $9.4 \mathrm{~cm}^{-1}$ for all bands except the monomer band (FWHM $=3.5 \mathrm{~cm}^{-1}$ ), a fit is shown in the inset of Fig. 4 for data obtained for deposition at $19 \mathrm{~K}$, noting that this case involves negligible tetramer contribution. We note the appearance of a shoulder at $2216.5 \mathrm{~cm}^{-1}$. This does not relate to any known $\mathrm{N}_{2} \mathrm{O}-\mathrm{N}_{2} \mathrm{O}$ interaction and may be due to an impurity. This shoulder is not found in spectra taken for higher temperature films.

Fits of the RAIR spectra with the contributions assigned to $\mathrm{N}_{2} \mathrm{O}$ monomer, dimer, trimer and tetramer can be adjusted to give good agreement at $20 \mathrm{~K}$ of deposition and up to $30 \mathrm{~K}$ of annealing. For deposition at $29 \mathrm{~K}$ and above however, a better fit may be obtained by including an additional contribution on the high wavenumber side. This contribution, composed of two peaks at 2234 and $2240 \mathrm{~cm}^{-1}\left(\right.$ FWHMs $\left.=9.4 \mathrm{~cm}^{-1}\right)$, most likely arises from associations (or 'clusters') larger than the tetramer. There remains however, as noted above, no contribution from a pure $\mathrm{N}_{2} \mathrm{O}$ phase since the characteristic doublet bands at 2260 and $2235 \mathrm{~cm}^{-1}$ are absent.

The area of the gaussians used to fit the RAIR spectra is taken to be proportional to the amount of $\mathrm{N}_{2} \mathrm{O}$ in each configuration, that is, monomer, dimer etc., on the assumption that the strength of the $\nu \mathrm{NN}$ band in each configuration is similar. To allow the comparison between data from experiments performed on films held at different temperatures, the areas were normalised to the total area of the $\nu \mathrm{NN}$ band for every deposition temperature. The resulting quantities, proportional to the number of $\mathrm{N}_{2} \mathrm{O}$ molecules in each configuration, are given in Fig. 5. This shows

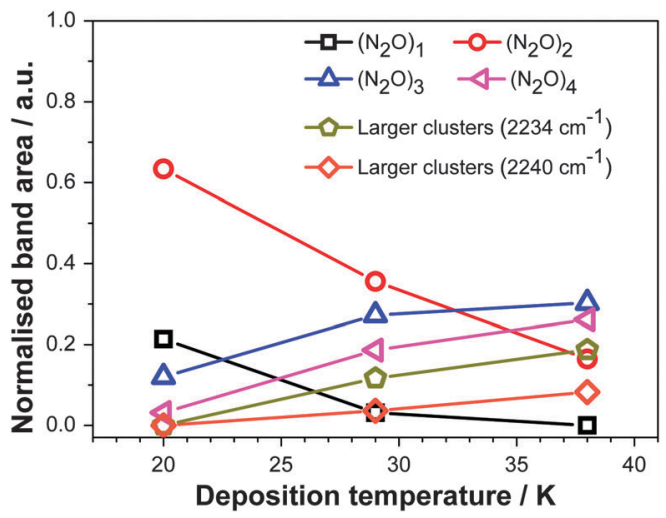

Fig. 5 Areas of the gaussian functions used to fit the RAIR spectra as a function of the deposition temperature for the $\xi=5$ films. The areas were normalized to the total area of the $\nu \mathrm{NN}$ band in the RAIR spectrum recorded at the same temperature to allow the comparison of different experiments with each other. Lines are a guide for the eye.

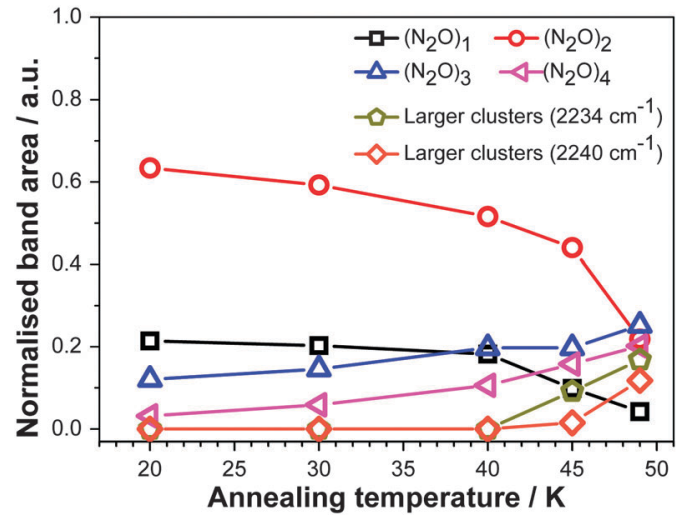

Fig. 6 Areas of the gaussian functions used to fit the RAIR spectra as a function of the annealing temperature for the $\xi=5$ films. The areas were normalized to the total area of the $\nu \mathrm{NN}$ band in the RAIR spectrum recorded at the same temperature. Lines are a guide for the eye.

the prominence of isolated $\mathrm{N}_{2} \mathrm{O}$ monomers and dimers in the $\xi=5$ mixtures deposited at $20 \mathrm{~K}$. Increasing the deposition temperature of the film leads to an increase of the amount of trimers, tetramers and larger associations at the expense of monomers and dimers - but not to the segregation of $\mathrm{N}_{2} \mathrm{O}$ from Xe. This reiterates the most significant result of the RAIRS data, that is, the absence of segregation. Fig. 6 shows equivalent data for annealing.

Our present results are consistent with the conclusions drawn in ref. 8 and 9 and extend these conclusions to much higher mixing ratios. $\mathrm{N}_{2} \mathrm{O}$ monomers, dimers, trimers and tetramers are observed to remain dispersed in the lattice, while a higher deposition temperature (or annealing) allows more association and cluster formation. The latter effect reflects the increased ability for mutual diffusion of Xe and $\mathrm{N}_{2} \mathrm{O}$ at higher temperatures but does not suggest any fundamental structural changes as the temperature of deposition is raised.

\section{A theoretical description}

\subsection{A model of diluted spontelectrics}

A mean-field model is used here to describe the spontelectric steady state. This model was originally introduced in ref. 4 and is set out in detail in ref. 1 . The model is based on the concept that the net $z$-component of the electric field within a spontelectric film and normal to the plane of the film, $E_{z}$, is composed of a local symmetrical part, defining the interactions which bind layers to one another, and an asymmetrical part due to the infinite range field which permeates the film. The symmetrical part is expressed as a constant term plus a dipole-dipole term, proportional to $\left(\left\langle\mu_{z}\right\rangle / \mu\right)^{2}$ and representing average intermolecular dipole-dipole interactions. Here $\left\langle\mu_{z}\right\rangle / \mu$, the degree of dipole orientation, is the ratio of the average $z$-component of the dipole moment and the total dipole moment of the molecular species in the solid state. The form of this term describing dipole-dipole interactions reflects the fact that all such interactions, involving dipole-image charge, extended dipoles and arrays of dipoles, follow a squared relation. ${ }^{17-19}$ The $\zeta\left(\left\langle\mu_{z}\right\rangle / \mu\right)^{2}$ term in eqn (1) below may be interpreted 
as a measure of the tendency of one dipolar species to restrict the angular motion of another, a 'locking' term or, as it is sometimes called, a 'frustration' term.

The symmetrical part of the contribution to $E_{z}$ is related to the 'local field' at any molecular site as defined in standard texts. ${ }^{20}$ The asymmetrical part, $\left\langle E_{\text {asym }}\right\rangle\left\langle\mu_{z}\right\rangle / \mu$, is found only in the description of spontelectrics and has no direct counterpart for any other form of material. This asymmetrical part however resembles the Weiss field in ferromagnetism, which is assumed to be proportional to the magnetisation. ${ }^{21}$ Here, read degree of dipole orientation for magnetisation and read polarisation field for the Weiss field. We emphasise that the polarisation field, that is, the spontelectric field, is self-generated within the spontelectric material and takes the place of an external field imposed on the film. The polarization field acts in opposition to the symmetrical part and represents the infinite range field created by the average dipoles and experienced by an average dipole.

Hence, using atomic units throughout,

$$
E_{z}=\left\langle E_{\mathrm{sym}}\right\rangle\left[1+\zeta\left(\frac{\left\langle\mu_{z}\right\rangle}{\mu}\right)^{2}\right]-\left\langle E_{\mathrm{asym}}\right\rangle \frac{\left\langle\mu_{z}\right\rangle}{\mu}
$$

where $\left\langle E_{\mathrm{sym}}\right\rangle,\left\langle E_{\text {asym }}\right\rangle$ and $\zeta$ are taken to be temperature independent parameters. Mean field theory gives an implicit expression for $\left\langle\mu_{z}\right\rangle / \mu$ yielding the familiar Langevin function for orientational interactions: ${ }^{20}$

$$
\frac{\left\langle\mu_{z}\right\rangle}{\mu}=\operatorname{coth}\left(\frac{E_{z} \mu}{T}\right)-\left(\frac{E_{z} \mu}{T}\right)^{-1}
$$

where $T$ is the deposition temperature of the layer of material. The dipole moment of $\mathrm{N}_{2} \mathrm{O}$ in the pure solid state is reduced from that in the gas phase through depolarization in the environment of other $\mathrm{N}_{2} \mathrm{O}$ species according to:

$$
\mu=\frac{\mu_{0}}{1+\alpha k / s_{\text {pure }}{ }^{3}}
$$

where $s_{\text {pure }}$ is the average spacing between successive layers $\left(=0.32 \mathrm{~nm}\right.$ for pure $\left.\mathrm{N}_{2} \mathrm{O}\right),{ }^{1,4} \alpha$ is the molecular polarizability of $\mathrm{N}_{2} \mathrm{O}\left(3.03 \times 10^{-30} \mathrm{~m}^{3}\right), k=11.034^{22}$ and $\mu_{0}$ is the gas phase dipole moment of $\mathrm{N}_{2} \mathrm{O}(=0.166 \mathrm{D})$.

This theoretical model is now adapted to cover the case in which $\mathrm{N}_{2} \mathrm{O}$ is diluted in Xe. The RAIRS data in Fig. 5 and 6 reveal the complication that associations of $\mathrm{N}_{2} \mathrm{O}$ of larger size increasingly form as the temperature is raised. However for simplicity we ignore any compositional changes associated with different temperatures and we describe the films as two component mixtures of Xe and an average $\mathrm{N}_{2} \mathrm{O}$ species, without explicit reference to temperature. Thus the composition of the film is treated as an average over the data between $38 \mathrm{~K}$ and $49 \mathrm{~K}$ in Fig. 6. We therefore derive below expressions for the variation of the parameter $\zeta$ (eqn (1)), the dipole moment of $\mathrm{N}_{2} \mathrm{O}$ and the degree of dipole orientation which contains only dilution dependence, effectively averaging over the temperature dependence of composition. The justification for this approach is that, though inexact, it turns out to give considerable insight into the nature of diluted spontelectrics, with a minimum of parameterization.

In keeping with the mean field approach which allows for only one type of active species, we treat the system of $\mathrm{N}_{2} \mathrm{O}$ configurations (monomers, dimers, timers etc.) as consisting of a set of randomly dispersed and identical $\mathrm{N}_{2} \mathrm{O}$ species. A dimer, $\left(\mathrm{N}_{2} \mathrm{O}\right)_{2}$ in Fig. 5 and 6 , is viewed as forming by a chance juxtaposition of two $\mathrm{N}_{2} \mathrm{O}$ monomers, a trimer, $\left(\mathrm{N}_{2} \mathrm{O}\right)_{3}$ in Fig. 5 and 6 , as a chance juxtaposition of three $\mathrm{N}_{2} \mathrm{O}$ monomers and so on. Pure $\mathrm{N}_{2} \mathrm{O}$ is the limit in which all $\mathrm{N}_{2} \mathrm{O}$ neighbours are themselves $\mathrm{N}_{2} \mathrm{O}$ molecules. Given this, the dipole moment of $\mathrm{N}_{2} \mathrm{O}$ in the solid mixture should be expected to increase towards the gas phase value for $\mathrm{N}_{2} \mathrm{O}$ as $\xi$ increases, on the assumption that Xe perturbs $\mathrm{N}_{2} \mathrm{O}$ only weakly. For lack of other information, and again in the interests of keeping the number of adjustable parameters to a minimum, $\mathrm{N}_{2} \mathrm{O}$ in its various mutual configurations is assumed to have the molecular volume and the related polarizability associated with $\mathrm{N}_{2} \mathrm{O}$ monomers.

On this basis, there are three interrelated parameters which need to be adjusted according to the experimental dilution of $\mathrm{N}_{2} \mathrm{O}$ in Xe. These are the average distance apart of the $\mathrm{N}_{2} \mathrm{O}$ species for a specific dilution, the value of $\zeta$ (eqn (1)), which determines the strength of the interaction between adjacent $\mathrm{N}_{2} \mathrm{O}$ species, and the value of the average dipole moment of $\mathrm{N}_{2} \mathrm{O}$ species relevant to the diluted solid mixtures, $\mu_{\xi}$, where the subscript $\xi$ refers throughout to the value of any parameter associated with a particular dilution.

Recollect here that we define the dilution in the film as the ratio of the number of atoms of Xe and of molecules of $\mathrm{N}_{2} \mathrm{O}$ condensed upon the $\mathrm{Au}$ substrate ratio, that is, $\xi=n_{\mathrm{Xe}} / n_{\mathrm{N}_{2} \mathrm{O}}$. The fraction of the total volume of the film taken up by $\mathrm{N}_{2} \mathrm{O}$ is then given by

$$
n_{\mathrm{N}_{2} \mathrm{O}} \Omega_{\mathrm{N}_{2} \mathrm{O}} /\left(n_{\mathrm{N}_{2} \mathrm{O}} \Omega_{\mathrm{N}_{2} \mathrm{O}}+n_{\mathrm{Xe}} \Omega_{\mathrm{Xe}}\right)
$$

and the total volume of the film taken up by Xe is given by

$$
n_{\mathrm{Xe}} \Omega_{\mathrm{Xe}} /\left(n_{\mathrm{N}_{2} \mathrm{O}} \Omega_{\mathrm{N}_{2} \mathrm{O}}+n_{\mathrm{Xe}} \Omega_{\mathrm{Xe}}\right)
$$

where $\Omega_{\mathrm{N}_{2} \mathrm{O}}$ is the volume taken up by an $\mathrm{N}_{2} \mathrm{O}$ molecule and $\Omega_{\text {Xe }}$ by a Xe atom in the solid film. In pure $\mathrm{N}_{2} \mathrm{O}$, the volume occupied by the film is given by $n_{\mathrm{N}_{2} \mathrm{O}} 4 / 3 \pi\left(r_{\mathrm{N}_{2} \mathrm{O}}\right)^{3}$ whereas, if diluted, the same number of molecules of $\mathrm{N}_{2} \mathrm{O}$ may be found in a volume $\xi n_{\mathrm{N}_{2} \mathrm{O}} \Omega_{\mathrm{Xe}}+$ $n_{\mathrm{N}_{2} \mathrm{O}} \Omega_{\mathrm{N}_{2} \mathrm{O}}$. Thus one molecule of $\mathrm{N}_{2} \mathrm{O}$ may be found in a volume of the solid film of $4 / 3 \pi r_{\xi}{ }^{3}$ for a film composed of a mixture of $\mathrm{N}_{2} \mathrm{O}$ and Xe with a dilution $\xi$. Hence $n_{\mathrm{N}_{2} \mathrm{O}} 4 / 3 \pi r_{\xi}{ }^{3}$, the total volume of the film, may be expressed as $\xi n_{\mathrm{N}_{2} \mathrm{O}} \Omega_{\mathrm{Xe}}+n_{\mathrm{N}_{2} \mathrm{O}} \Omega_{\mathrm{N}_{2} \mathrm{O}}$ which yields

$$
r_{\xi}=\left(\xi r_{\mathrm{Xe}}^{3}+r_{\mathrm{N}_{2} \mathrm{O}}^{3}\right)^{1 / 3}
$$

From this it follows the average distance apart of two $\mathrm{N}_{2} \mathrm{O}$ molecules, $2 r_{\xi}$, previously $s_{\text {pure }}$ (eqn (3)), but now $s_{\xi}$, is given by $2\left(\xi r_{\mathrm{Xe}}{ }^{3}+r_{\mathrm{N}_{2} \mathrm{O}}{ }^{3}\right)^{1 / 3}$. The lattice constant of pure solid Xe on a FCC lattice yields $r_{\mathrm{Xe}}=0.219 \mathrm{~nm}$ and, for pure $\mathrm{N}_{2} \mathrm{O}, r_{\mathrm{N}_{2} \mathrm{O}}$ has been estimated to be $0.16 \mathrm{~nm},{ }^{4}$ giving values of $s_{\xi}$ as a function of $\xi$.

We turn now to the evaluation of $\zeta_{\xi}$. The potential between two dipoles, at an angle $\theta$ to one another, is given by $-\mu_{\xi}{ }^{2} / 8 \varepsilon r_{\xi}^{3}\left(1-3 \cos ^{2} \theta\right)$ where $\varepsilon$ is some permittivity. The latter 
quantity, at the low temperatures encountered here, should to a good approximation be independent of $\xi$. It then follows that $\zeta_{\xi} / \zeta_{\mathrm{N}_{2} \mathrm{O}}$, that is, the ratio of the value of $\zeta$ in the mixture of monomers, dimers, trimers etc. of $\mathrm{N}_{2} \mathrm{O}$ and that in the pure state, is given by

$$
\zeta_{\xi} / \zeta_{\mathrm{N}_{2} \mathrm{O}}=r_{\mathrm{N}_{2} \mathrm{O}}^{3} \mu_{\xi}^{2} / r_{\xi}^{3} \mu_{\mathrm{N}_{2} \mathrm{O}}{ }^{2}
$$

where $\mu_{\mathrm{N}_{2} \mathrm{O}}$ is the dipole moment of $\mathrm{N}_{2} \mathrm{O}$ in the pure solid state and $\mu_{\xi}$ is defined above. The variation with $\xi$ of the term multiplying $\left\langle E_{\text {sym }}\right\rangle$ in eqn (1) will therefore be given by a product of the varying values of $\zeta_{\xi}\left(\left\langle\mu_{z}\right\rangle / \mu\right)_{\xi}{ }^{2}$ with the corresponding values of $\left\langle E_{\mathrm{sym}}\right\rangle_{\xi}$. Since the parameter $\left\langle E_{\mathrm{sym}}\right\rangle_{\xi}$ is associated with polarisation, dispersion and covalent forces, we do not attempt to estimate the variation of this term with $\xi$. Instead $\left\langle E_{\text {sym }}\right\rangle_{\xi}$ remains as a variable parameter in subsequent fitting of theory to experiment.

Using eqn (3), which relates the solid state dipole moment to the gas phase value, with eqn (4) and inserting $s_{\xi}=2 r_{\xi}=2\left(\xi r_{\mathrm{Xe}}^{3}+r_{\mathrm{N}_{2} \mathrm{O}}^{3}\right)^{1 / 3}$ derived above, we obtain

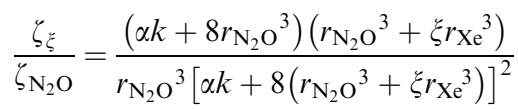

where $\alpha$ and $k$ are defined above in connection with eqn (3). Implicit in eqn (5) is that the variation of the average effective dipole moment of $\mathrm{N}_{2} \mathrm{O}$ in $\mathrm{Xe}$ is given by inserting $s_{\xi}=2\left(\xi r_{\mathrm{Xe}}{ }^{3}+r_{\mathrm{N}_{2} \mathrm{O}}{ }^{3}\right)^{1 / 3}$ in eqn (3) to yield

$$
\mu_{\xi}=\frac{\mu_{0}}{\left[1+\alpha k / 8\left(\xi r_{\mathrm{Xe}^{3}}+r_{\mathrm{N}_{2} \mathrm{O}^{3}}\right)\right]}
$$

Values of the separation of $\mathrm{N}_{2} \mathrm{O}$ molecules, $s_{\xi}$, the observed electric field based upon this value of $s_{\xi}$, and of $\zeta_{\xi}$ are shown in Table 4 , both for the three different temperatures of $\mathrm{N}_{2} \mathrm{O}$ Xe mixtures investigated, $38 \mathrm{~K}, 44 \mathrm{~K}$ and $48 \mathrm{~K}$, and for different dilutions at $44 \mathrm{~K}$ and $48 \mathrm{~K}$. Parameters for pure $\mathrm{N}_{2} \mathrm{O}(\xi=0)$ are included for comparison, for which values were obtained from a fit between theory and experiment reported in ref. 1 and 4 .

Values in Table 4 reflect our intuition that the value of $\zeta_{\xi}$ drops with increasing dilution, $\xi$, in the limit that, as dilution tends to infinity, so dipole-dipole interactions tend to zero. Again the increasing average distance between $\mathrm{N}_{2} \mathrm{O}$ molecules, associated with increasing dilution, creates a dipole moment in the solid which approaches that in the gas phase of 0.0684 au $(0.167 \mathrm{D})$, as indicated through eqn (6). This incidentally exposes an additional hidden assumption that the presence of Xe perturbs negligibly the electronic structure of $\mathrm{N}_{2} \mathrm{O}$, an assumption supported for example by results in ref. 9. In this connection one may note that although Xe is a highly polarizable atom, with $\alpha=4.01 \times 10^{-30} \mathrm{~m}^{3}$, it can readily be shown that the dipole generated in Xe by the presence of $\mathrm{N}_{2} \mathrm{O}$ is only of the order of $0.002 \mathrm{D}$, making a negligibly small contribution to the field at $\mathrm{N}_{2} \mathrm{O}$.
Table 4 The variation of $s_{\xi}, \zeta_{\xi}, E_{\text {obs }}$ and $\mu_{\xi}$ for both temperature and dilution based upon experimental observations of the spontelectric potential (Tables 1-3 and Fig. 1-3) and equations given in the text. Note that to obtain the dipole moment in Debyes, divide the value in au by 0.409353

\begin{tabular}{llllll}
\hline Temperature $/ \mathrm{K}$ & $E_{\mathrm{obs}} / \mathrm{V} \mathrm{m}$ & $\xi=n_{\mathrm{Xe}} / n_{\mathrm{N}_{2} \mathrm{O}}$ & $s_{\xi} / \mathrm{nm}$ & $\zeta_{\xi}$ & $\mu_{\xi} / \mathrm{au}$ \\
\hline 38 & $1.189 \times 10^{8}$ & Zero & 0.320 & 43.8 & 0.03214 \\
44 & $7.889 \times 10^{7}$ & Zero & 0.320 & & \\
48 & $6.616 \times 10^{7}$ & Zero & 0.320 & & \\
& & & & & \\
38 & $3.032 \times 10^{7}$ & 2.1 & 0.594 & 22.23 & 0.05785 \\
44 & $2.769 \times 10^{7}$ & 2.2 & 0.602 & 21.62 & 0.05819 \\
48 & $1.899 \times 10^{7}$ & 2.8 & 0.645 & 18.54 & 0.05980 \\
& & & & & \\
44 & $3.026 \times 10^{7}$ & 1.0 & 0.489 & 31.89 & 0.05176 \\
44 & $6.230 \times 10^{6}$ & 26 & 1.304 & 2.801 & 0.06685 \\
44 & Zero & 67 & 1.782 & 1.119 & 0.06752 \\
& & & & & \\
48 & $3.407 \times 10^{7}$ & 0.9 & 0.477 & 33.12 & 0.05083 \\
48 & $1.899 \times 10^{7}$ & 2.8 & 0.645 & 18.54 & 0.05980 \\
48 & $5.378 \times 10^{6}$ & 23 & 1.253 & 3.147 & 0.06671 \\
& & & & &
\end{tabular}

\subsection{Comparison of model and experimental values}

3.2.1 Variation of temperature with approximately constant dilution. The purpose of this section is to use the above model to fit the three values of electric field for $38 \mathrm{~K}, 44 \mathrm{~K}$ and $48 \mathrm{~K}$, with respective dilutions, $\xi$, of 2.1, 2.2 and 2.8 (Table 3) using the parameters set out in Table 4 . Recollect for this purpose that (i) the total column densities of nitrous oxide are close to being equal in all three cases, respectively $N=240 \mathrm{ML}, 245 \mathrm{ML}$ and $227 \mathrm{ML}$ and (ii) the electric field, $E_{\text {obs, }}$, is given by the measured voltage on the surface of the film, $V$, divided by the estimated thickness of the film, $N s_{\xi}$.

Results in Table 5 give the values of parameters required exactly to reproduce the values of electric field, $E_{\mathrm{obs}}$, in rows $4-6$ of Table 4 (also shown in Table 5 for convenience). This is achieved by varying only the value of $\left\langle E_{\mathrm{sym}}\right\rangle$, where $\left\langle E_{\mathrm{sym}}\right\rangle$, or more properly $\left\langle E_{\mathrm{sym}}\right\rangle_{\xi}$, is defined in connection with eqn (1). Recollecting that $\left\langle E_{\text {asym }}\right\rangle_{\xi}=$ $\mu_{\xi} / \varepsilon_{0} \Omega$ where $\Omega$ is the molecular volume $\left(=37.79 \AA^{3}\right)^{1}$ a value of the degree of dipole orientation $\left\langle\mu_{z}\right\rangle / \mu_{\xi}$ follows, since $E_{\text {obs }}=\left\langle E_{\text {asym }}\right\rangle_{\xi}\left\langle\mu_{z}\right\rangle /$ $\mu_{\xi}$. As seen in Table 5 , values of $\left\langle\mu_{z}\right\rangle / \mu_{\xi}$, corresponding to the values of the fitting parameter $\left\langle E_{\mathrm{sym}}\right\rangle_{\xi}$, lie between 0.01 to 0.02 with a dilution of 2 to 3 , very much less than the values for the pure material $^{1}$ of 0.08 to 0.15 shown in the last column of Table 5 . In connection with values in Table $5, E_{\text {obs }}$ is found to be only very weakly sensitive to variation of the value of $\zeta_{\xi}$ which have therefore been held at the values computed from eqn (5) and given in Table 4.

Table 5 Values of the parameter $\left\langle E_{\text {sym }}\right\rangle_{\xi}$ used to reproduce the experimental values of $E_{\text {obs }}$ (also given) as function of temperature of deposition for similar dilution parameters, $\xi$, as shown. Corresponding values of the

\begin{tabular}{|c|c|c|c|c|c|}
\hline Temperature/K & $E_{\mathrm{obs}} / \mathrm{V} \mathrm{m}^{-1}$ & $\xi$ & $\left\langle E_{\mathrm{sym}}\right\rangle_{\xi} / \mathrm{V} \mathrm{m}{ }^{-1}$ & $\begin{array}{l}\text { Diluted } \\
\mathrm{N}_{2} \mathrm{O}\left\langle\mu_{z}\right\rangle / \mu_{\xi}\end{array}$ & $\begin{array}{l}\text { Pure } \mathrm{N}_{2} \mathrm{O} \\
\left\langle\mu_{z}\right\rangle / \mu\end{array}$ \\
\hline 38 & $3.032 \times 10^{7}$ & 2.1 & $1.252 \times 10^{8}$ & 0.0207 & 0.1552 \\
\hline 44 & $2.769 \times 10^{7}$ & 2.2 & $1.094 \times 10^{8}$ & 0.0188 & 0.0981 \\
\hline 48 & $1.899 \times 10^{7}$ & 2.8 & $8.128 \times 10^{7}$ & 0.0125 & 0.0826 \\
\hline
\end{tabular}
degree of dipole orientation are also shown, with values for pure $\mathrm{N}_{2} \mathrm{O}$ taken from ref. 1 and 4 for comparison 
3.2.2 Variation of dilution at constant deposition temperature. The purpose here is to fit data for $E_{\text {obs }}$ at $44 \mathrm{~K}$ and $48 \mathrm{~K}$ (rows 7-12 of Table 4) for different dilutions for similar column densities of $\mathrm{N}_{2} \mathrm{O}$. Once more it turns out to be possible exactly to reproduce the experimental value of electric field, $E_{\mathrm{obs}}$ in Table 3 , by varying only the value of $\left\langle E_{\text {sym }}\right\rangle_{\xi}$. The resulting values of $\left\langle E_{\mathrm{sym}}\right\rangle_{\xi}$ and $\left\langle\mu_{z}\right\rangle / \mu_{\xi}$ are shown in Table 6 , with the value of the degree of dipole orientation for pure $\mathrm{N}_{2} \mathrm{O}$ given in the last column for comparison.

In connection with results in Tables 4-6, we note that for the pure material it proved possible to fit the variation of the observed spontelectric field with temperature of deposition with a single value of $\left\langle E_{\mathrm{sym}}\right\rangle=5.43 \pm 0.2 \times 10^{8} \mathrm{~V} \mathrm{~m}^{-1} .^{1,4}$ Results in Table 5 tend to confirm this for a diluted sample; the relatively small variation in $\left\langle E_{\mathrm{sym}}\right\rangle_{\xi}$ may be put down to the variation in values of dilution, $\xi$, encountered in these experiments. Results in Table 6 however show a large drop in $\left\langle E_{\text {sym }}\right\rangle_{\xi}$ as dilution is increased. This reflects the property that atoms of Xe create a much weaker local field than that created by dipoledipole, polarisation, dispersion and covalent forces between molecules of $\mathrm{N}_{2} \mathrm{O}$.

3.2.3 Power law variations of the degree of dipole orientation with dilution. Values for $\left\langle\mu_{z}\right\rangle / \mu_{\xi}$ presented in Table 6 show that this quantity may be represented by a power law in the dilution $\xi$, omitting the case of $\xi=67$ for $44 \mathrm{~K}$. Taking data for $48 \mathrm{~K}$ as an example, we find that

$$
\left\langle\mu_{z}\right\rangle / \mu_{\xi} \sim \kappa \xi^{-\lambda}
$$

where $\kappa=0.025$ and $\lambda=0.65$ in the range $0.9 \leq \xi \leq 23$. It may be shown, again empirically, that $\mu_{\xi}\left\langle E_{\mathrm{sym}}\right\rangle_{\xi}$ may be represented by a power law in $\xi$ of the form

$$
\mu_{\xi}\left\langle E_{\mathrm{sym}}\right\rangle_{\xi} \sim \sigma \xi^{-\tau}
$$

for the same range of $\xi$, where $\sigma=1.51 \times 10^{-5}, \tau=0.45$ and $\mu_{\xi}$ is given by eqn (6). Note that $\mu_{\xi}\left\langle E_{\mathrm{sym}}\right\rangle_{\xi}$ is an energy, which is related to the depth of the well restricting molecular rotation in the film (see Section 4).

Recollecting that values of $\left\langle\mu_{z}\right\rangle / \mu_{\xi}$ and $\mu_{\xi}\left\langle E_{\text {sym }}\right\rangle_{\xi}$ are themselves derived from a model represented by eqn (1) to (6) above, our purpose here is to show that the relationships (7) and (8) are as far as possible in algebraic agreement with the model

Table 6 Values of the parameter $\left\langle E_{\text {sym }}\right\rangle_{\xi}$ used to reproduce the experimental values of $E_{\text {obs }}$ (also in Table 4) as function of dilution, $\xi$, as shown. Corresponding values of the degree of dipole orientation are also shown, with values for pure $\mathrm{N}_{2} \mathrm{O}$ taken from ref. 1 and 4 for comparison. Note that $E_{\mathrm{obs}}=0$ for $\xi=67$ at a deposition temperature of $44 \mathrm{~K}$

\begin{tabular}{llllll}
\hline & $\begin{array}{l}E_{\text {obs }} / \\
\text { Temperature } / \mathrm{K} \mathrm{V}^{-1}\end{array}$ & $\xi$ & $\begin{array}{l}\left\langle E_{\text {sym }}\right\rangle_{\xi} / \\
\mathrm{V} \mathrm{m}^{-1}\end{array}$ & $\begin{array}{l}\text { Diluted } \\
\mathrm{N}_{2} \mathrm{O}\left\langle\mu_{z}\right\rangle / \mu_{\xi}\end{array}$ & $\begin{array}{l}\text { Pure } \mathrm{N}_{2} \mathrm{O} \\
\left\langle\mu_{z}\right\rangle / \mu\end{array}$ \\
\hline 44 & $3.026 \times 10^{7}$ & 1 & $1.405 \times 10^{8}$ & 0.0231 & 0.0981 \\
44 & $2.769 \times 10^{7}$ & 2.2 & $1.094 \times 10^{8}$ & 0.0188 & \\
44 & $6.230 \times 10^{6}$ & 26 & $3.117 \times 10^{7}$ & 0.0037 & \\
44 & Zero & 67 & $1.381 \times 10^{7}$ & 0 & \\
& & & & & \\
48 & $3.407 \times 10^{7}$ & 0.9 & $1.602 \times 10^{8}$ & 0.0265 & 0.0826 \\
48 & $1.899 \times 10^{7}$ & 2.8 & $8.128 \times 10^{7}$ & 0.0125 & \\
48 & $5.378 \times 10^{6}$ & 23 & $2.808 \times 10^{7}$ & 0.0032 &
\end{tabular}

embodied in eqn (1) to (6). This acts as a check on the selfconsistency of our analysis.

We now introduce two approximations both of which may be justified numerically in the present case. The first is that $\operatorname{coth}(x)-1 / x=x / 3$ in eqn (2) and the second derives from an algebraic analysis, based on the first approximation, which shows that the degree of dipole orientation $\left\langle\mu_{z}\right\rangle / \mu_{\xi}$ may be expressed as $d_{3} d_{4} / 2 d_{2}$ where

$$
\begin{gathered}
d_{1}=r_{\mathrm{N}_{2} \mathrm{O}}{ }^{3}+\xi r_{\mathrm{Xe}}{ }^{3} \\
d_{2}=256 \pi \mu_{0}^{2} d_{1}^{2}+3 T \Omega\left(8 d_{1}+\alpha k\right)^{2} \\
d_{3}=4 \sigma^{2} d_{1} \zeta_{\xi} \Omega^{2} \xi^{-2 \tau}\left(8 d_{1}+\alpha k\right)^{2}\left(8 r_{\mathrm{N}_{2} \mathrm{O}}{ }^{3}+\alpha k\right)^{2} / r_{\mathrm{N}_{2} \mathrm{O}}{ }^{3} \\
d_{4}=r_{\mathrm{N}_{2} \mathrm{O}}{ }^{3} \xi^{\tau} /\left[2 \sigma d_{1} \zeta \xi \Omega\left(8 r_{\mathrm{N}_{2} \mathrm{O}}{ }^{3}+\alpha k\right)^{2}\right]
\end{gathered}
$$

given that numerically $d_{3} / d_{2}^{2} \ll 1$.

We then find that:

$$
\left\langle\mu_{z}\right\rangle / \mu_{\xi}=\mu_{\xi}\left\langle E_{\mathrm{sym}}\right\rangle_{\xi} /\left[3 T\left(1+\frac{256 \pi \mu_{0}^{2} d_{1}^{2}}{3 T \Omega\left(\alpha k+8 d_{1}\right)^{2}}\right)\right]
$$

We need now to show that the rhs of eqn (9) can be written in the form $\kappa \xi^{-\lambda}$ as found by fitting to experimental values (eqn (7)). However we do not know, save from experiment, how $\left\langle E_{\text {sym }}\right\rangle_{\xi}$ varies with $\xi$, that is essentially how the field in the environment of an average $\mathrm{N}_{2} \mathrm{O}$ species depends on the degree of dilution of $\mathrm{N}_{2} \mathrm{O}$ in Xe. To proceed, we therefore introduce the observation that $\mu_{\xi}\left\langle E_{\mathrm{sym}}\right\rangle_{\xi}$ can be replaced by $\sigma \xi^{-\tau}$, eqn (8), noting also that $d_{1}$ in eqn (9) contains $\xi$. We therefore obtain:

$$
\left\langle\mu_{z}\right\rangle / \mu_{\xi}=\sigma \xi^{-\tau} /\left[3 T\left(1+\frac{256 \pi \mu_{0}^{2}\left(r_{\mathrm{N}_{2} \mathrm{O}}{ }^{3}+\xi r_{\mathrm{Xe}}{ }^{3}\right)^{2}}{3 T \Omega\left[\alpha k+8\left(r_{\mathrm{N}_{2} \mathrm{O}}{ }^{3}+\xi r_{\mathrm{Xe}}\right)^{2}\right]^{2}}\right)\right]
$$

This shows that the variation of $\left\langle\mu_{z}\right\rangle / \mu_{\xi}$ with dilution, $\xi$, indeed follows an exponential form, as required by eqn (7), but modified by the denominator in eqn (10).

For eqn (7) and (8) to be consistent as desired, it should be possible approximately to represent eqn (10) by $\kappa \xi^{-\lambda}$ such that $\kappa \sim 0.0246$ and $\lambda \sim 0.6533$ when $\sigma \sim 1.51 \times 10^{-5}$ and $\tau \sim 0.45$, as derived empirically. If for example we hold $\kappa$ and $\lambda$ at the empirical values and allow $\sigma$ and $\tau$ to vary, we find that for $\sigma=1.45 \times 10^{-5}$ and $\tau=0.59$ we obtain $\left\langle\mu_{z}\right\rangle / \mu_{\xi} v s$. $\xi$ in better than $1 \%$ agreement at low dilution rising to $\sim 6.5 \%$ for $\xi=23$. Alternatively, consistency of eqn (7) and (8) can for example be achieved within $3 \%$ for all values of $\xi$ by varying all four parameters, $\kappa, \lambda, \sigma$ and $\tau$ with $\kappa=0.028$ and $\lambda=0.6$ when $\sigma=1.43 \times 10^{-5}$ and $\tau=0.5$.

This brief analysis demonstrates that the parameterization introduced intuitively into the theoretical model, in particular in developing eqn (5) and (6) for $\zeta_{\xi}$ and $\mu_{\xi}$, is consistent with the observed exponentially decreasing behaviour of the degree of dipole orientation with dilution. 


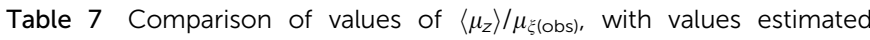
from eqn (9), $\left\langle\mu_{z}\right\rangle / \mu_{\xi(\text { (calc). }}$ See eqn (7) and (8) (Section 3.2.3) for definitions of $\sigma$ and $\tau$

\begin{tabular}{llllll}
\hline Temperature $/ \mathrm{K}$ & $\xi$ & $\sigma$ & $\tau$ & $\left\langle\mu_{z}\right\rangle / \mu_{\xi(\mathrm{calc})}$ & $\left\langle\mu_{z}\right\rangle / \mu_{\xi(\mathrm{obs})}$ \\
\hline 38 & 2.1 & $1.52 \times 10^{-5}$ & 0.350 & 0.0224 & 0.0207 \\
44 & 2.2 & $1.53 \times 10^{-5}$ & 0.400 & 0.0191 & 0.0188 \\
48 & 2.8 & $1.51 \times 10^{-5}$ & 0.453 & 0.0149 & 0.0125
\end{tabular}

Eqn (9) can also be used for comparison with data for the temperature dependence of the degree of dipole orientation for a similar value of $\xi$. This is shown in Table 7 . For $38 \mathrm{~K}$, an average value of $\sigma$ for $44 \mathrm{~K}$ and $48 \mathrm{~K}$ has been used and a smaller value of $\tau$ has been estimated, referring to parameters in eqn (7) and (8). Results for $\left\langle\mu_{z}\right\rangle / \mu_{\xi \text { (calc) }}$ are insensitive to these choices.

3.2.4 The high dilution case: absence of the spontelectric effect. Table 1 shows that at $44 \mathrm{~K}$ for a dilution of 67 , that is, $\xi=67$, no electric field can be detected in the solid film. This is a significant result of the present work, since it leads directly to the conclusion that some mutual degree of restriction of rotational motion is essential to the many body collective physics which leads to the formation of the spontelectric state. We show below that the lack of detection of an electric field at high dilution is not due to lack of sensitivity of the system but is a real result lying well within the capabilities of the experimental system.

As shown in Table $4, \zeta_{\xi}$ has been estimated to fall to 1.119 at $\xi=67$. This may be compared with the value of $\zeta_{\xi}=2.801$ for the $\xi=26$ or $\zeta_{\xi}=43.8$ in the pure material. Thus we suggest that somewhere between these two figures of $\zeta_{\xi}$ of 1.119 and 2.801 , the strength of the dipole-dipole interaction becomes sufficiently weak, at $44 \mathrm{~K}$ deposition temperature, that collective dipole orientation becomes unattainable. In this connection, the increase in the strength of the dipole is negligible, lying at $1 \%$ over the range of the layer spacing $s_{\xi}$ of $1.304 \mathrm{~nm}$ for $\xi=26$ to $1.782 \mathrm{~nm}$ for $\xi=67$ (Table 4).

To substantiate the absence of a spontelectric field, it is now shown, on the basis of the theoretical description given above, that the observed electric field for $\xi=67$ should be quite substantial and a potential should readily be measured, if the material were spontelectric in the manner described for lower dilutions. First we note that the observed spontelectric field, $E_{\text {obs }}$, is given by the second term in eqn (1), that is, $\left\langle E_{\text {asym }}\right\rangle\left\langle\mu_{z}\right\rangle / \mu$, (see Section 4.1 of ref. 1) and thus:

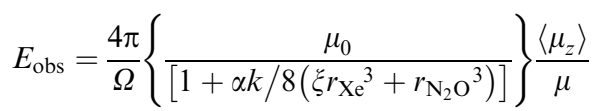

Using atomic units, $4 \pi / \Omega=0.04928$ and $\alpha k=246.466$ and inserting values of $\mu_{0}, r_{\mathrm{Xe}}(0.219 \mathrm{~nm})$ and $r_{\mathrm{N}_{2} \mathrm{O}}(0.16 \mathrm{~nm})$, we obtain:

$$
E_{\mathrm{obs}}=0.003348 \frac{\left\langle\mu_{z}\right\rangle}{\mu} /(1+0.3127 / \xi)
$$

where for simplicity we have used $\xi r_{\mathrm{Xe}}{ }^{3} \gg r_{\mathrm{N}_{2} \mathrm{O}}{ }^{3}$ introducing an error of $4 \%$ for $\xi=67$. Thus for $\xi=67, E_{\text {obs }}$ should take the value $0.0033\left\langle\mu_{z}\right\rangle / \mu$ or $1.7 \times 10^{9}\left\langle\mu_{z}\right\rangle / \mu \mathrm{V} \mathrm{m}^{-1}$. For $44 \mathrm{~K}$, experimental data in Table 4 show that $\left\langle\mu_{z}\right\rangle / \mu=0.0258 \xi^{-0.585}$, yielding a value of dipole orientation of 0.0022 for $\xi=67$, a figure which may be compared with 0.0037 for $\xi=26$ at $44 \mathrm{~K}$ in Table 6 . Thus if the material remained spontelectric at $\xi=67$, one should measure an electric field of $3.74 \times 10^{6} \mathrm{~V} \mathrm{~m}^{-1}$.

Experiments have for example been performed for a total column density of $175 \mathrm{ML}$ of $\mathrm{N}_{2} \mathrm{O}$ equivalent to a total film thickness of $175 \times 1.782=312 \mathrm{~nm}$ (see Table $2,44 \mathrm{~K}, \xi=67$ ). This would yield a potential on the surface of $\sim 1.16 \mathrm{~V}$ given a field of $3.74 \times 10^{6} \mathrm{~V} \mathrm{~m}^{-1}$. We find a measurement of zero within experimental error, whereas if the material remained spontelectric at this dilution, the above estimate shows that a value in excess of $1 \mathrm{~V}$ should be observed. Hence the lack of detection of a spontelectric effect at $\xi=67$ clearly shows that the spontelectric effect is switched off at sufficiently high dilution.

\section{Concluding remarks}

At the outset two questions were posed whose answers had as their object the provision of greater insight into the nature of the spontelectric state. These questions were (i) how does the degree of dipole orientation change as the spontelectric species are removed physically from one another? (ii) Is there some physical separation between species or species dilution at which the spontelectric effect can no longer be observed?

With respect to the first question, we find that dipole orientation is reduced on dilution of $\mathrm{N}_{2} \mathrm{O}$ in Xe. Thus for the same column density of material, a smaller spontaneous electric field is generated when the $\mathrm{N}_{2} \mathrm{O}$ molecules are positioned on average further apart. With respect to the second, we find that given sufficient dilution, the spontelectric effect is no longer present.

There are two factors at play here in the context of both the questions posed and the answers given. First, we recollect that the dipole term in eqn (1), $\left\langle E_{\mathrm{sym}}\right\rangle \zeta\left(\left\langle\mu_{z}\right\rangle / \mu\right)^{2}$ may be interpreted as a measure of the tendency of one dipole to restrict the angular motion of another. The first term of eqn (1), simply $\left\langle E_{\text {sym }}\right\rangle$, also effectively attempts to hold the dipole in a vice-like grip, that is, from both sides, restricting any form of motion. The relative importance of these two terms, $\left\langle E_{\text {sym }}\right\rangle$ and $\left\langle E_{\text {sym }}\right\rangle \zeta\left(\left\langle\mu_{z}\right\rangle / \mu\right)^{2}$, is strongly dependent on the degree of dilution. For example at zero dilution the dipole-dipole interaction is $30 \%$ (at $48 \mathrm{~K}$ ) to $45 \%$ (at $44 \mathrm{~K}$ ) of the total symmetric field $\left\langle E_{\text {sym }}\right\rangle\left\lfloor 1+\zeta\left(\left\langle\mu_{z}\right\rangle / \mu\right)^{2}\right\rfloor$, whereas at high dilution, $\xi=23$ to 26 , this reduces to $0.003 \%$ to $0.004 \%$ (and, at $\xi=67$, to zero at $44 \mathrm{~K})$. At the same time the total symmetrical field falls by a factor of $\sim 25$ from between 7.13-7.85 $\times 10^{8} \mathrm{~V} \mathrm{~m}^{-1}$ for pure $\mathrm{N}_{2} \mathrm{O}^{1}$ to $2.8-3 \times 10^{7} \mathrm{~V} \mathrm{~m}^{-1}$ for $\xi=23$ to 26 , where figures span the range $48 \mathrm{~K}$ to $44 \mathrm{~K}$. This reflects both the drop in dipoledipole interactions and the weaker interaction between $\mathrm{N}_{2} \mathrm{O}$ with Xe, compared with $\mathrm{N}_{2} \mathrm{O}$ with itself, as dilution is increased. This is itself accompanied by an increase in effective dipole moment in the solid by a factor of $\sim 2.1$, resulting in a net decrease in $\left\langle E_{\mathrm{sym}}\right\rangle_{\xi} \cdot \mu_{\xi}$ by a factor of $\sim 12$ on dilution from pure $\mathrm{N}_{2} \mathrm{O}$ to $\xi=23-26$. Thus in diluted mixtures of $\mathrm{N}_{2} \mathrm{O}$ and Xe, the $\mathrm{N}_{2} \mathrm{O}$ molecules sit in a well which restricts rotation whose depth 
is an order of magnitude smaller at $\xi=23$ to 26 compared with pure $\mathrm{N}_{2} \mathrm{O}$ or a factor of $\sim 5$ between $\xi=0.9$ to 1 and 23 to 26 .

At high dilution, rotational motion is therefore much less restricted and thermal energy can overpower $\left\langle E_{\mathrm{sym}}\right\rangle_{\xi} \cdot \mu_{\xi}$. For example for $\xi=26$ at $44 \mathrm{~K},\left\langle E_{\mathrm{sym}}\right\rangle_{\xi} \cdot \mu_{\xi}$ is $\sim 3 \%$ of $k T$ whereas the figure for $\xi=1$ is $\sim 10 \%$ and for pure $\mathrm{N}_{2} \mathrm{O}$ is $\sim 25 \%$. Given that average dipole orientation in the steady state is in part related to competition between random thermal motion and the restriction of rotational motion, it is therefore evident why $\left\langle\mu_{z}\right\rangle / \mu$ falls to small values at high dilution. For $\xi=67$ for $44 \mathrm{~K},\left\langle E_{\mathrm{sym}}\right\rangle_{\xi} \cdot \mu_{\xi}$ is $\sim 1.3 \%$ of $k T$ indicating that at some point where the $\left\langle E_{\mathrm{sym}}\right\rangle_{\xi} \cdot \mu_{\xi}$ electrical interaction lies between 1 and $3 \%$ of $k T$, the spontelectric effect is altogether inhibited. The conclusion is that a local field of sufficient strength to restrict rotation is necessary to the development of the non-local many body collective physics associated with spontelectrics and that the local field-dipole interaction must be at least 1 to $3 \%$ of $k T$. This is not reflected in the theoretical description of spontelectrics presented here, which makes the assumption that a spontelectric state may always be achieved. However it sets a goal for molecular dynamics simulations of the spontelectric effect.

Second, results may be understood in the context of the model involving fluctuating motions of dipoles, put forward in the introduction, to illustrate how the spontelectric state may form dynamically. Some fluctuation, whose average physical extent depends on the temperature of deposition and the degree of dilution, leads to local dipole orientation $\left\langle\mu_{z}\right\rangle / \mu$, which we note will be in the opposite sense to that encountered in the spontelectric state. A fluctuation is therefore able to create a fleeting polarisation, $\left(\left\langle\mu_{z}\right\rangle / \mu\right)_{\xi} \cdot\left(\mu_{\xi} / \Omega\right)$, and thus a field will briefly arise. We suggest that the more dilute the material the less the value of dipole orientation which will be formed through fluctuations. This arises because of the greater freedom of rotational movement associated with greater dilution, as described above in relation to the spontelectric state but equally applicable here. The compensating field, the spontelectric field, will be correspondingly less as dilution is increased.

\section{Acknowledgements}

We gratefully acknowledge support of the staff of the Aarhus Synchrotron Radiation Laboratory (ISA), the Danish Research Council, a Marie Curie Intra-European Fellowship 009786 (RB), the European Community FP7-ITN Marie-Curie Programme (LASSIE project, grant agreement \#238258) (AC, JL), the Lundbeck Foundation (RB) and Heriot-Watt University for a James Watt scholarship (ARF).

\section{Notes and references}

1 D. Field, O. Plekan, A. Cassidy, R. Balog, N. C. Jones and J. Dunger, Int. Rev. Phys. Chem., 2013, 32, 345-392.

2 R. Balog, P. Cicman, N. Jones and D. Field, Phys. Rev. Lett., 2009, 102, 2-5.

3 D. Field, O. Plekan, A. Cassidy, R. Balog and N. Jones, Europhys. News, 2011, 42, 32-35.

4 O. Plekan, A. Cassidy, R. Balog, N. C. Jones and D. Field, Phys. Chem. Chem. Phys., 2011, 13, 21035-21044.

5 O. Plekan, A. Cassidy, R. Balog, N. C. Jones and D. Field, Phys. Chem. Chem. Phys., 2012, 14, 9972-9976.

6 A. Cassidy, O. Plekan, R. Balog, N. C. Jones and D. Field, Phys. Chem. Chem. Phys., 2012, 15, 108-113.

7 A. Cassidy, O. Plekan, R. Balog, J. Dunger, N. C. Jones and D. Field, J. Phys. Chem. A, 2014, 118, 6615-6621, DOI: 10.1021/jp503332x.

8 H. Krueger and E. Weitz, J. Chem. Phys., 1992, 96, 2846.

9 W. G. Lawrence and V. A. Apkarian, J. Chem. Phys., 1992, 97, 2224.

10 H. J. Fraser, M. P. Collings and M. R. S. McCoustra, Rev. Sci. Instrum., 2002, 73, 2161.

11 A. Łapiński, J. Spanget-Larsen, J. Waluk and J. G. Radziszewski, J. Chem. Phys., 2001, 115, 1757.

12 D. Fulvio, B. Sivaraman, G. A. Baratta, M. E. Palumbo and N. J. Mason, Spectrochim. Acta, Part A, 2009, 72, 1007-1013.

13 The $\nu \mathrm{NN}$ band of the pure $\mathrm{N}_{2} \mathrm{O}$ multilayer is split into longitudinal and transverse modes in RAIR spectra. Both modes are visible when the spectra are recorded at non-normal incidence and the film is deposited on a non-metallic substrate, ${ }^{11,12}$ as is the case here. The behaviour of these LO and TO bands has been thoroughly investigated and will be presented in a separate publication on the nature of the spontelectric effect in pure $\mathrm{N}_{2} \mathrm{O}$.

14 S. Kudoh, K. Onoda, M. Takayanagi and M. Nakata, J. Mol. Struct., 2000, 524, 61-68.

15 J. Sodeau and R. Withnall, J. Phys. Chem., 1985, 89, 4484-4488. 16 J. N. Oliaee, M. Dehghany, N. Moazzen-Ahmadi and A. R. W. McKellar, J. Chem. Phys., 2011, 134, 074310.

17 E. Cohen de Lara and J. Vincent-Geisse, J. Phys. Chem., 1976, 80, 1922-1927.

18 B. L. Maschhoff and J. P. Cowin, J. Chem. Phys., 1994, 101, 8138.

19 D. Fernández-Torre, O. Kupiainen, P. Pyykkö and L. Halonen, Chem. Phys. Lett., 2009, 471, 239-243.

20 C. Kittel, Introduction to Solid State Physics, Wiley, 3rd edn, 2005.

21 H. Kliem, M. Kuehn and B. Martin, Ferroelectrics, 2010, 400, 41-51.

22 J. Topping, Proc. R. Soc. London, Ser. A, 1927, 114, 67-72. 